\title{
Expansão da vegetação arbustivo-arbórea em áreas abertas alto-montanas adjacentes a fragmentos florestais, no Planalto Sul Catarinense
}

\author{
Expansion of tree-shrub vegetation in upper-montane open areas adjacent to forest fragments, in \\ "Planalto Sul Catarinense" region
}

\author{
Juliana Pizutti Dallabrida ${ }^{\mathrm{I}}$, Ana Carolina da Silva ${ }^{\text {II }}$, Pedro Higuchi ${ }^{\mathrm{II}}$, Janaina Gabriela Larsen ${ }^{\mathrm{III}}$, \\ Guilherme Neto Santos ${ }^{\mathrm{III}}$, Carla Luciane Lima ${ }^{\mathrm{I}}$, Luiz Carlos Rodrigues Júnior ${ }^{\mathrm{III}}$, \\ Felipe Domingos Machado ${ }^{\mathrm{III}}$, Aline Pereira Cruz ${ }^{\mathrm{IV}}$, Amanda da Silva Nunes ${ }^{\mathrm{I}}$
}

\begin{abstract}
Resumo
O objetivo deste estudo foi conhecer os padrões demográficos da regeneração natural do componente arbustivo-arbóreo e determinar se esses são influenciados pelas variáveis ambientais, em três áreas abertas em regeneração natural (diâmetro na altura do peito $<5 \mathrm{~cm}$ ), em contato com fragmentos florestais de Floresta Ombrófila Mista Alto-Montana, no Planalto Sul Catarinense. Foram instaladas três transecções, uma por área, de 20 x $100 \mathrm{~m}$, subdividas em subparcelas de $10 \times 10 \mathrm{~m}$, perpendiculares à borda dos fragmentos, com sua extensão cobrindo a área de campo. Amostraram-se todos os indivíduos regenerantes arbustivo-arbóreos com $20 \mathrm{~cm}$ ou mais de altura. Em cada subparcela, foram coletados os seguintes dados ambientais: propriedades físico-químicas dos solos, grau de compactação dos solos e cota altimétrica. Um ano após o inventário, foram obtidas as taxas de dinâmica para as três comunidades e para as populações, e os padrões encontrados foram relacionados com as variáveis ambientais. Os dados foram analisados por meio de testes de Kruskal-Wallis, testes de comparações múltiplas, teste de qui-quadrado aplicado à tabela de contingência, teste de correlação, Análise de Componentes Principais (PCA) e modelos lineares mistos. Os resultados demonstraram que, no período estudado, o processo de regeneração natural do componente arbustivo-arbóreo encontra-se em fase inicial e foi caracterizado pelo incremento na abundância de indivíduos, estabilidade na riqueza, independência espacial entre a mortalidade e o recrutamento e limitação do recrutamento em função da baixa fertilidade natural do solo e elevada disponibilidade de Al.
\end{abstract}

Palavras-chave: Floresta Ombrófila Mista Alto-Montana; Padrões de dinâmica; Análise ambiental

\begin{abstract}
The study aimed to understand the dynamic patterns of natural regeneration of shrub-tree species and determine whether these patterns are influenced by environmental variables, in three open areas in natural regeneration (diameter at breast height $<5 \mathrm{~cm}$ ), in contact to upper-montane Araucaria Forest fragments, located in the Southern Plateau of Santa Catarina state. Three 20x100 m transects, subdivided into sub-plots of 10x10 m, were installed in each area, perpendicular to the edge of the fragments with their length covering the grassland area. All shrub-tree regenerating individuals greater than or equal to $20 \mathrm{~cm}$ height were sampled. In each sub-plot, the environmental data were collected: soil physicochemical properties, the degree of soil compaction and the altimetric quota. One year after the first inventory, the dynamics rates were obtained for the three community and populations, and the found patterns related to environmental variables. The data were analyzed through Kruskal-Wallis tests, multiple comparison tests, chi-square test, applied to a contingency table, Principal Component Analysis (PCA) and linear mixed models. The results demonstrated that during the evaluated period, the natural regeneration process of the shrub-tree component is found in an early stage and it was characterized by abundance increment, richness stability, spatial independency between mortality and recruitment, and recruitment limitation in function of low soil natural fertility and high Al availability.
\end{abstract}

Keywords: Highlands Araucaria Forest; Dynamic patterns; Environmental analysis

\footnotetext{
I Engenheira Florestal, MSc., Departamento de Engenharia Florestal, Universidade do Estado de Santa Catarina, Av. Luiz de Camões, 2090, CEP 88520-000, Lages (SC), Brasil.juli_ajuri@hotmail.com (ORCID: 0000-0002-9766-8714) / carla_engflorestal@yahoo.com.br(ORCID: 0000-0002-7943-3962) / amandaeng.f@gmail.com (ORCID: 0000-0001-6669-3936)

II Engenheira(o) Florestal, Dr., Professor do Departamento de Engenharia Florestal, Universidade do Estado de Santa Catarina, Av. Luiz de Camões, 2090, CEP 88520-000, Lages (SC), Brasil. carol_sil4@yahoo.com.br (ORCID: 0000-0002-1285-640X)/ higuchip@gmail.com (ORCID: 0000-0002-3855-555X)

III Engenheiro Florestal, Departamento de Engenharia Florestal, Universidade do Estado de Santa Catarina, Av. Luiz de Camões, 2090, CEP 88520-000, Lages (SC), Brasil. janainalarsen18@gmail.com (ORCID: 0000-0002-5603-5208)/ guineto.florestal@gmail.com (ORCID: 00000003-0346-6941)/ luiz.crj@hotmail.com (ORCID: 0000-0001-7270-9009)/ felipe.d.machado@hotmail.com (ORCID: 0000-0002-1731-4730)

${ }^{\text {IV }}$ Bióloga, MSc., Departamento de Engenharia Florestal, Universidade do Estado de Santa Catarina, Av. Luiz de Camões, 2090, CEP 88520-000, Lages (SC), Brasil. a.line_cruz@yahoo.com.br (ORCID: 0000-0002-9279-7293)
} 


\section{Introdução}

A Floresta Ombrófila Mista (FOM) Alto-Montana faz parte de um mosaico formado por florestas e áreas de vegetação aberta, constituídas por campos naturais (Estepes), considerados como uma vegetação proveniente de períodos glaciais do Quaternário, mais secos e frios (BEHLING et al., 2007; BEHLING; PILLAR, 2007; SILVA; ANAND, 2011), e por áreas desmatadas para dar origem a atividades antrópicas. De acordo com Jeske-Pieruschka et al. (2013), nos locais em que as condições edáficas são favoráveis, o clima atual da região sul favorece a expansão do componente florestal sobre as áreas abertas, sendo este processo limitado pelas ações antropogênicas desde o Holoceno Tardio.

Atualmente, as perturbações ocasionadas pela utilização do fogo e pelo pastejo podem ser consideradas como importantes controladores da expansão da floresta sobre as áreas abertas no sul Brasil (MÜLLER et al., 2007; OVERBECK et al., 2007). Assim, quando se deseja observar a dinâmica da expansão florestal sem a influência do manejo, o ideal é estudar áreas com exclusão de fogo e pastejo, como as áreas avaliadas do presente estudo, que foram desapropriadas para a formação do Parque Nacional de São Joaquim e, atualmente, encontram-se protegidas. Segundo Oliveira e Pillar (2004), o processo de expansão tende a ocorrer em bordas de florestas contínuas, o que também justifica a escolha das áreas estudadas, adjacentes à fragmentos florestais.

Alguns estudos sobre a expansão da vegetação florestal têm sido realizados no sul Brasil, como o de Oliveira e Pillar (2004), que observaram maior avanço em áreas onde o campo apresentava abundância de indivíduos de Baccharis uncinella DC., que podem promover o sombreamento. No entanto, ainda existem lacunas a serem preenchidas em relação ao conhecimento dos padrões de expansão da vegetação arbórea sobre áreas abertas, especialmente em relação à influência de características edáficas e topográficas sobre as taxas demográficas da vegetação. Desta forma, este trabalho teve por objetivo conhecer a dinâmica da regeneração natural de espécies arbustivo-arbóreas em áreas de vegetação aberta alto-montanas localizadas no Parque Nacional de São Joaquim, Santa Catarina, e determinar as variáveis ambientais influentes nos padrões encontrados. A hipótese é de que a expansão da vegetação arbustivo-arbórea, sintetizada pelas suas taxas demográficas, seja influenciada pelas condições edáficas e topográficas.

\section{Material e métodos}

O estudo foi realizado em três áreas de vegetação aberta, adjacentes a fragmentos florestais, no Parque Nacional de São Joaquim. O parque tem sua sede no município de Urubici, no qual foram alocadas as unidades amostrais. O clima da região, segundo classificação de Köppen, é do tipo Cfb e, de acordo com a base de dados do Worldclim (HIJMANS et al., 2005), a precipitação média anual é $1.753 \mathrm{~mm}$ e a temperatura média anual é $12,7^{\circ} \mathrm{C}$. A vegetação é formada por um mosaico de campos e florestas, sendo as florestas classificadas como FOM Alto-Montana, segundo o IBGE (2012). Assim, as áreas de floresta formam fragmentos naturais na paisagem, existindo também fragmentos antrópicos, formados pela exploração madeireira e criação de gado. Os solos predominantes são classificados como Neossolos Litólicos e Cambissolos; e o relevo varia de suave a fortemente ondulado.

Com o propósito de investigar o processo de ocupação florestal nas diferentes condições campestres comuns no Sul do Brasil, a amostragem contemplou regenerantes arbustivo-arbóreos ocorrentes tanto em campos naturais, quanto em campos antrópicos. A primeira área inventariada (Área 1), localizada a $28^{\circ} 05^{\prime} 41,5^{\prime}$ 'S $, 49^{\circ} 30^{\prime} 14,71$ ' $\mathrm{W}$ e com altitude de $1.628 \mathrm{~m}$, era de floresta e foi desmatada na década de 1960, sendo utilizada para pastagem, com utilização do fogo. Ela foi desapropriada em 2007 e atualmente está protegida. A segunda área (Área 2), localizada a $28^{\circ} 04^{\prime} 46,87^{\prime}$ 'S, $49^{\circ} 30^{\prime} 51,29^{\prime \prime} \mathrm{W}$ e com altitude de $1.356 \mathrm{~m}$, era de floresta e foi desmatada para exploração madeireira na década de 1980 e, desde essa época, foi manejada com o uso do fogo e criação de gado. Foi desapropriada em 2008 e também está protegida. A terceira área (Área 3), localizada a $28^{\circ} 09^{\prime} 49,19^{\prime \prime}$ S, $49^{\circ} 36^{\prime} 47,56^{\prime \prime} \mathrm{W}$ e com altitude de $1.660 \mathrm{~m}$, é campo (Estepe) desde os tempos remotos, com gado desde o século XIX. Foi desapropriada e protegida em 2008.

Em julho de 2014 foram instaladas três transecções, uma em cada área, de largura de $20 \mathrm{~m}$ por 100 m de comprimento, subdivida em subparcelas de 10 x $10 \mathrm{~m}$. Cada transecção contou com 20 subparcelas 
e teve $2.000 \mathrm{~m}^{2}$ de área total. As transecções foram alocadas perpendiculares à borda dos fragmentos, com sua extensão cobrindo a área aberta. Foram amostrados, plaqueteados e identificados todos os indivíduos regenerantes pertencentes a espécies arbustivo-arbóreas dentro das parcelas com $20 \mathrm{~cm}$ ou mais de altura. O estudo não considerou indivíduos arbóreos adultos (DAP - diâmetro a altura do peito - maior ou igual a $5 \mathrm{~cm}$ ) porque esses eram escassos nas áreas (considerando as três áreas, são menos que 10 indivíduos). As identificações botânicas foram realizadas com o auxílio de especialistas (Dr. Marcos Eduardo Guerra Sobral e Dr. Cláudio Augusto Mondin) e literatura, de forma que foi possível ter segurança de que todos os indivíduos coletados pertenciam às formas de vida consideradas no presente estudo (arbustos e árvores), e as coletas foram incorporadas ao Herbário LUSC, da Universidade do Estado de Santa Catarina. Em julho de 2015, um ano após a primeira coleta de dados, as áreas foram revisitadas para a realização do estudo da dinâmica dos regenerantes, sendo todos os indivíduos recontados para verificação da mortalidade e do recrutamento e identificação daqueles que atingiram a altura mínima de $20 \mathrm{~cm}$. As taxas de mortalidade (TxMor), recrutamento (TxRec), mudança líquida em número de indivíduos (TxML) e rotatividade em número de indivíduos (TxRot) foram calculadas para cada uma das comunidades e para as populações pelas fórmulas utilizadas por Korning e Balslev (1994), Sheil, Jennings e Savill (2000) e Oliveira Filho et al. (2007): TxMor $=(1-((\mathrm{N} 1-\mathrm{NMor}) / \mathrm{N} 1) 1 / \mathrm{t}) \times 100 ;$ TxRec $=(1-(1-\mathrm{NRec} / \mathrm{N} 2) 1 / \mathrm{t}) \times 100 ; \mathrm{TxML}=((\mathrm{N} 2 /$ N1) $1 / t-1) \times 100$; TxRot $=($ TxMor+TxRec $) / 2$; onde: $\mathrm{N} 1=$ número inicial de indivíduos; Nmor $=$ número de indivíduos mortos; $\mathrm{t}=$ tempo; $\mathrm{Nrec}=$ número de indivíduos recrutas; $\mathrm{N} 2$ = número final de indivíduos.

As espécies amostradas em ambos os anos foram classificadas em guildas de regeneração, segundo a metodologia proposta por Swaine e Whitmore (1988) e adaptada por Oliveira Filho et al. (1994b), em: pioneira (P), clímax exigente em luz (CEL) e clímax tolerante ao sombreamento (CTS), com base nas observações a campo e consulta à literatura.

Foi realizada a caracterização ambiental das áreas pela obtenção das variáveis edáficas e topográficas. As propriedades físico-químicas dos solos foram determinadas a partir da coleta de nove amostras no perfil de 0 a $20 \mathrm{~cm}$, em cada uma das 60 subparcelas. As nove coletas foram homogeneizadas, retirando-se uma amostra composta por subparcela. As análises das propriedades físico-químicas dos solos foram realizadas na Universidade do Estado de Santa Catarina (UDESC), quantificando-se: teores de areia, silte e argila, $\mathrm{pH}$ em $\mathrm{H}_{2} \mathrm{O}$, cálcio, magnésio, alumínio, sódio, fósforo, potássio, capacidade de troca catiônica (CTC efetiva), soma de bases e matéria orgânica. O grau de compactação do solo foi obtido por meio de testes de resistência à penetração vertical no solo, utilizando um penetrômetro de impacto digital modelo Penetrolog PLG1020 Falker, com o cone tipo 2, com dez repetições sistemáticas em cada parcela, na profundidade de 0 a $20 \mathrm{~cm}$. Dessas dez leituras, foi retirada uma média por parcela. Dessa forma, em cada parcela foi obtida uma leitura de resistência à penetração a $1 \mathrm{~cm}, 2 \mathrm{~cm}$, até $20 \mathrm{~cm}$ de profundidade. Considerou-se, na caracterização da resistência à penetração em cada parcela, a média dessas leituras de $1 \mathrm{~cm}$ até $20 \mathrm{~cm}$, a resistência à penetração máxima nesse perfil e a resistência à penetração somente a 20 $\mathrm{cm}$ de profundidade. No levantamento topográfico, três variáveis topográficas foram determinadas em cada parcela: cota, desnível máximo e declividade média. A cota foi calculada pela média das cotas dos quatro vértices da parcela, o desnível máximo correspondeu à maior distância vertical entre os vértices da parcela (OLIVEIRA FILHO et al., 1994a) e a declividade foi calculada pela média da declividade dos quatro lados da parcela.

As taxas demográficas (mortalidade, recrutamento, rotatividade e mudança líquida), calculadas por meio das fórmulas indicadas acima, foram comparadas entre as áreas por meio do teste de KrukalWallis, uma vez que os dados não apresentaram distribuição normal, seguido por um teste de comparação múltipla não paramétrico. A associação entre os padrões de dinâmica (incremento, estabilidade ou redução do tamanho populacional) das espécies e as guildas de regeneração para as espécies das áreas 1 e 2 (área 3 não testada, em função da baixa abundância de regenerantes arbustivo-arbóreos) foi verificada por meio de um teste de qui-quadrado aplicado a uma tabela de contingência. A relação entre as taxas demográficas foi analisada por meio de testes de correlação. A análise da heterogeneidade ambiental entre as parcelas de cada área foi realizada por meio de uma Análise de Componentes Principais (PCA), tendo como base as variáveis ambientais amostradas nas parcelas. A influência dos gradientes ambientais sobre as taxas demográficas foi analisada por meio de modelos lineares mistos, uma vez que as taxas demográficas das parcelas de uma mesma área tendem a ser mais semelhantes entre si, devido às diferenças de histórico de uso, estágio sucessional e de altitude. Desta forma, o eixo da PCA que explicou a maior proporção da 
variação dos dados ambientais foi considerado como efeito fixo, e as diferentes áreas, como efeito aleatório. A área 3 não foi incluída na modelagem, uma vez que apresentou poucas parcelas com indivíduos, o que representaria uma fonte de inconsistências na análise. Foram ajustados modelos considerando diferentes estruturas de efeito aleatório (intercepto e intercepto+coeficientes angulares). Quando as variâncias não apresentaram homogeneidade ao longo dos valores ajustados e ao longo das variáveis explicativas, foram adicionados aos modelos, funções para incorporar a estrutura da heterocedasticidade existente para cada área (ZUUR et al., 2009). Ainda, quando necessário, foi aplicada a transformação logarítmica junto às variáveis dependentes, com o propósito de estabilizar a variância e a aproximação da normalidade dos resíduos. A seleção e avaliação do modelo final se deu a partir do critério de AIC e análise da normalidade e homocedasticidade dos resíduos (ZUUR et al., 2009). As análises foram realizadas por meio da linguagem de programação estatística R (R DEVELOPMENT CORE TEAM, 2015).

\section{Resultados}

No período de estudo, foram observadas mudanças em número de indivíduos e tendência de estabilidade na riqueza de espécies nas áreas avaliadas, com a Área 2 apresentando maior proporção de espécies e indivíduos pioneiros do que a Área 1 (Tabela 1). A Área 3 também apresentou elevada proporção de espécies e indivíduos iniciais de sucessão, no entanto, com reduzido número de indivíduos amostrados. Apesar das três áreas apresentarem incremento na abundância em número de indivíduos, destaca-se o maior equilíbrio entre mortalidade e recrutamento na Área 2 (área de maior mortalidade), o que resultou em uma menor mudança líquida neste local $\left(5,17 \%\right.$.ano $\left.{ }^{-1}\right)$, quando comparado com os outros (Área $1=21,16 \%$.ano ${ }^{-1}$; Área $3=33,30 \%$.ano ${ }^{-1}$ ) $($ Tabela 2 ).

Tabela 1 - Número e porcentagem (\%) de espécies (Ne) e indivíduos (Ni) nas guildas de regeneração, em cada uma das três áreas estudadas no Parque Nacional de São Joaquim, SC.

Table 1 - Number and percentage (\%) of species (Ne) and trees (Ni) in regeneration guilds, in each of studied area, at São Joaquim National Park, SC state.

\begin{tabular}{|c|c|c|c|c|c|c|}
\hline & & \multicolumn{4}{|c|}{ Guildas de regeneração } & \multirow{2}{*}{ Tota } \\
\hline & & $\mathbf{P}$ & CEL & CTS & NC & \\
\hline \multirow[t]{4}{*}{ Área 1} & Ne 2014 & $9(32,14 \%)$ & $12(42,86 \%)$ & $5(17,86 \%)$ & $2(7,14 \%)$ & 28 \\
\hline & Ne 2015 & $9(31,03 \%)$ & $12(41,38 \%)$ & $5(17,24 \%)$ & $3(10,34 \%)$ & 29 \\
\hline & Ni 2014 & $\begin{array}{c}484 \\
(70,14 \%)\end{array}$ & $\begin{array}{c}151 \\
(21,88 \%)\end{array}$ & $48(6,96 \%)$ & $7(1,01 \%)$ & 690 \\
\hline & Ni 2015 & $\begin{array}{c}601 \\
(71,89 \%)\end{array}$ & $\begin{array}{c}174 \\
(20,81 \%)\end{array}$ & $53(6,34 \%)$ & $8(0,96 \%)$ & 836 \\
\hline \multirow{4}{*}{ Área 2} & Ne 2014 & $11(44,00 \%)$ & $9(36,00 \%)$ & $1(4,00 \%)$ & $4(16,00 \%)$ & 25 \\
\hline & Ne 2015 & $11(44,00 \%)$ & $10(40,00 \%)$ & $1(4,00 \%)$ & $3(12,00 \%)$ & 25 \\
\hline & Ni 2014 & $\begin{array}{c}618 \\
(88,79 \%)\end{array}$ & $60(8,62 \%)$ & $10(1,44 \%)$ & $8(1,15 \%)$ & 696 \\
\hline & Ni 2015 & $\begin{array}{c}644 \\
(87,98 \%) \\
\end{array}$ & $66(9,02 \%)$ & $10(1,37 \%)$ & $12(1,64 \%)$ & 732 \\
\hline \multirow{4}{*}{ Área 3} & $\mathrm{Ne} 2014$ & $2(66,67 \%)$ & $1(33,33 \%)$ & $0(0,00 \%)$ & $0(0,00 \%)$ & 3 \\
\hline & Ne 2015 & $3(75,00 \%)$ & $1(25,00 \%)$ & $0(0,00 \%)$ & $0(0,00 \%)$ & 4 \\
\hline & Ni 2014 & $3(50,00 \%)$ & $3(50,00 \%)$ & $0(0,00 \%)$ & $0(0,00 \%)$ & 6 \\
\hline & Ni 2015 & $5(62,50 \%)$ & $3(37,50 \%)$ & $0(0,00 \%)$ & $0(0,00 \%)$ & 8 \\
\hline
\end{tabular}

Em que: $\mathrm{P}=$ pioneira; $\mathrm{CEL}=$ clímax exigente em luz; $\mathrm{CTS}=$ clímax tolerante à sombra;

$\mathrm{NC}=$ não classificada. 
Tabela 2 - Dinâmica das espécies arbustivo-arbóreas regenerantes em três áreas (A1, A2 e A3) estudadas no Parque Nacional de São Joaquim, SC.

Table 2 - Dynamics of regenerating tree-shrub species in three studied areas (A1, A2 and A3) at São Joaquim National Park, SC state.

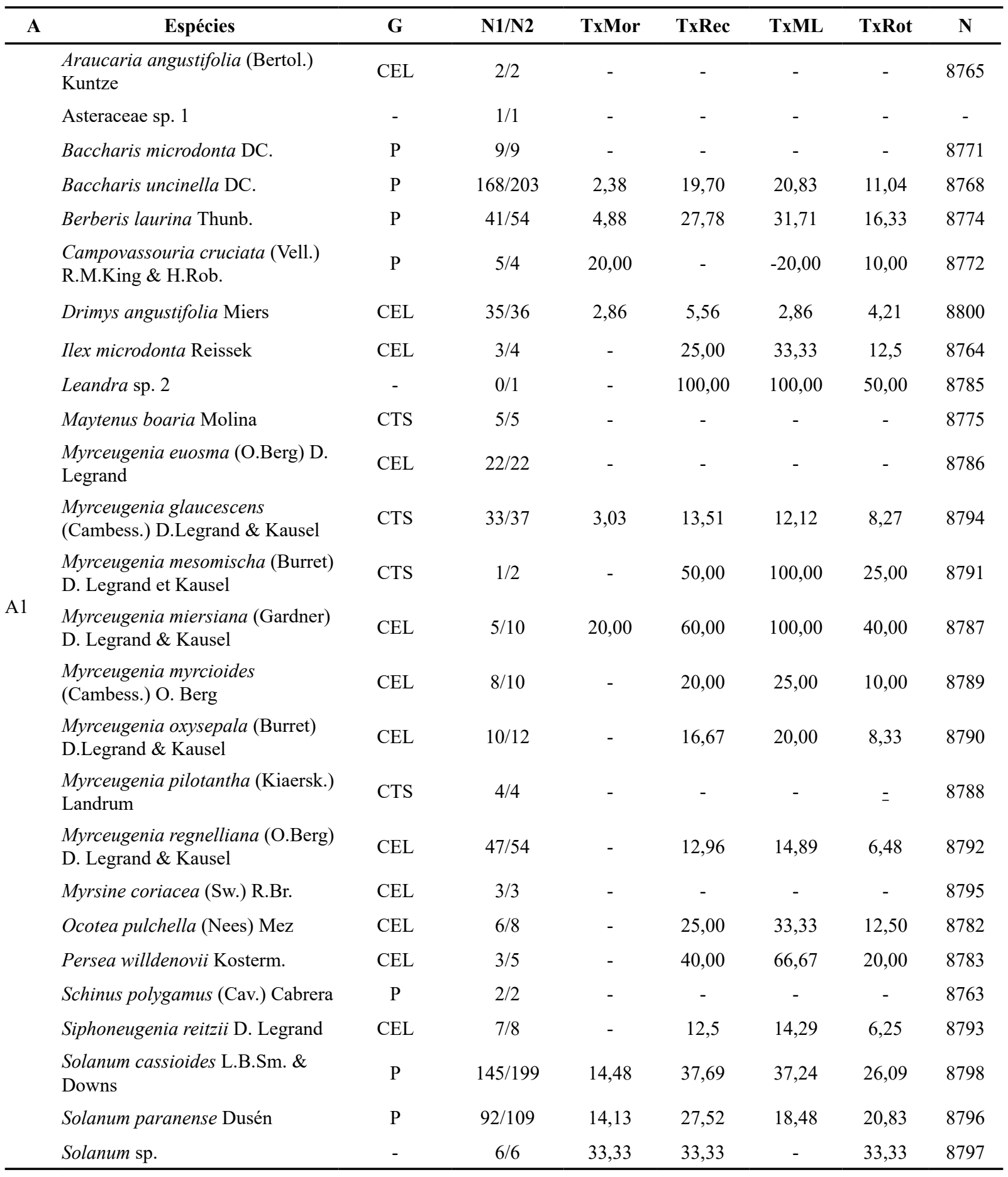


Tabela 2 - Continuação...

Table 2 - Continued...

\begin{tabular}{|c|c|c|c|c|c|c|c|c|}
\hline $\mathbf{A}$ & Espécies & G & N1/N2 & TxMor & TxRec & TxML & TxRot & $\mathbf{N}$ \\
\hline \multirow{4}{*}{ A1 } & $\begin{array}{l}\text { Symphyopappus itatiayensis } \\
\text { (Hieron.) R.M.King \& H.Rob. }\end{array}$ & $\mathrm{P}$ & $19 / 18$ & 10,53 & 5,56 & $-5,26$ & 8,04 & 8773 \\
\hline & Symplocos pentandra Occhioni & CTS & $5 / 5$ & - & - & - & - & 8799 \\
\hline & $\begin{array}{l}\text { Vernonanthura montevidensis } \\
\text { (Spreng.) H.Rob. }\end{array}$ & $\mathrm{P}$ & $3 / 3$ & - & - & - & - & 8770 \\
\hline & Total Área 1 & & $690 / 836$ & $6,96^{\mathrm{b}}$ & 23,33 & 21,16 & $15,14^{\mathrm{b}}$ & \\
\hline \multirow{22}{*}{ A2 } & Araucaria angustifolia & CEL & $7 / 7$ & - & - & - & - & 8765 \\
\hline & Baccharis microdonta & $\mathrm{P}$ & $10 / 27$ & 40,00 & 77,78 & 170,00 & 58,89 & 8771 \\
\hline & Baccharis uncinella & $\mathrm{P}$ & $188 / 184$ & 7,98 & 5,98 & $-2,13$ & 6,98 & 8768 \\
\hline & Berberis laurina & $\mathrm{P}$ & $27 / 30$ & 3,7 & 13,33 & 11,11 & 8,52 & 8774 \\
\hline & $\begin{array}{l}\text { Buddleia reitzii E.M. Norman } \\
\text { \& L.B. Sm. }\end{array}$ & $\mathrm{P}$ & $2 / 2$ & - & - & - & - & 8777 \\
\hline & Campovassouria cruciata & $\mathrm{P}$ & $143 / 173$ & 13,29 & 28,32 & 20,98 & 20,81 & 8772 \\
\hline & $\begin{array}{l}\text { Cinnamomum aтоепит Nees } \\
\text { (Kosterm.) }\end{array}$ & CEL & $10 / 11$ & 10,00 & 9,09 & 10,00 & 9,55 & 8781 \\
\hline & Clethra uleana Sleumer & CEL & $1 / 1$ & - & - & - & - & 8776 \\
\hline & $\begin{array}{l}\text { Croton cf. patrum L. B. Sm. \& } \\
\text { Downs }\end{array}$ & $\mathrm{P}$ & $92 / 62$ & 47,83 & 22,58 & $-32,61$ & 35,2 & 8762 \\
\hline & Drimys angustifolia & CEL & $3 / 3$ & - & - & - & - & 8800 \\
\hline & Escallonia bifida Link \& Otto & $\mathrm{P}$ & $4 / 4$ & - & - & - & - & 8779 \\
\hline & Leandra sp. 1 & - & $5 / 7$ & 60,00 & 71,43 & 40,00 & 65,71 & 8784 \\
\hline & Leandra sp. 2 & - & $2 / 2$ & - & - & - & - & 8785 \\
\hline & Mimosa pilulifera Benth. & $\mathrm{P}$ & $1 / 1$ & - & - & - & - & 8780 \\
\hline & Mimosa scabrella Benth. & $\mathrm{P}$ & $11 / 11$ & 9,09 & - & - & 4,55 & - \\
\hline & Myrceugenia euosma & CEL & $17 / 17$ & - & - & - & - & 8786 \\
\hline & Myrceugenia glaucescens & CTS & $10 / 10$ & - & - & - & - & 8794 \\
\hline & Myrceugenia myrcioides & CEL & $0 / 1$ & - & 100,00 & 100,00 & 50,00 & 8789 \\
\hline & Myrceugenia oxysepala & CEL & $3 / 4$ & - & 25,00 & 33,33 & 12,50 & 8790 \\
\hline & Myrceugenia regnelliana & CEL & $10 / 10$ & - & - & - & - & 8792 \\
\hline & Myrsine coriacea & CEL & $8 / 11$ & 12,50 & 36,36 & 37,50 & 24,43 & 8795 \\
\hline & $\begin{array}{l}\text { Senecio brasiliensis (Spreng.) } \\
\text { Less. }\end{array}$ & CEL & $105 / 96$ & 62,86 & 59,38 & $-8,57$ & 61,12 & 8766 \\
\hline \multirow{5}{*}{ A3 } & Siphoneugenia reitzii & CEL & $1 / 1$ & - & - & - & - & 8793 \\
\hline & Solanum paranense & $\mathrm{P}$ & $25 / 47$ & 8,00 & 51,06 & 88,00 & 29,59 & 8796 \\
\hline & Solanum sp. & - & $1 / 0$ & 100,00 & - & $-100,00$ & 50,00 & 8797 \\
\hline & Vernonanthura montevidensis & $\mathrm{P}$ & $10 / 10$ & - & - & - & - & 8770 \\
\hline & Total Área 2 & & $696 / 73$ & $22,70^{\mathrm{a}}$ & 26,37 & 5,17 & 24,53 & \\
\hline
\end{tabular}


Tabela 2 - Conclusão...

Table 2 - Conclusion...

\begin{tabular}{|c|c|c|c|c|c|c|c|c|}
\hline $\mathbf{A}$ & Espécies & $\mathbf{G}$ & $\mathbf{N} 1 / \mathbf{N} 2$ & TxMor & TxRec & TxML & TxRot & $\mathbf{N}$ \\
\hline \multirow{4}{*}{ A3 } & $\begin{array}{l}\text { Baccharis nummularia Heering ex } \\
\text { Malme }\end{array}$ & $P$ & $0 / 2$ & - & 100,00 & 100,00 & 50,00 & 8769 \\
\hline & Baccharis uncinella & $\mathrm{P}$ & $2 / 2$ & - & - & - & - & 8768 \\
\hline & Gaylussacia angustifolia Cham. & $\mathrm{P}$ & $1 / 1$ & - & - & - & - & 8778 \\
\hline & Siphoneugenia reitzii & $\begin{array}{c}\mathrm{CE} \\
\mathrm{L}\end{array}$ & $3 / 3$ & - & - & - & - & 8793 \\
\hline \multicolumn{2}{|r|}{ Total Área 3} & & $6 / 8$ & $0^{\mathrm{b}}$ & 25,00 & 33,30 & $12,50^{\mathrm{ab}}$ & \\
\hline
\end{tabular}

Em que: $\mathrm{G}$ = guildas de regeneração $(\mathrm{P}$ - pioneira; $\mathrm{CEL}$ - clímax exigente em luz; CTS - clímax tolerante ao sombreamento); N1 = número de indivíduos em 2014; N2 = número de indivíduos em 2015; TxMor = taxa de mortalidade $\left(\%\right.$.ano $\left.{ }^{-1}\right)$; TxRec $=$ taxa de recrutamento $\left(\%\right.$.ano $\left.{ }^{-1}\right)$; $\mathrm{TxML}=$ taxa de mudança líquida em número de indivíduos $\left(\% \cdot \mathrm{ano}^{-1}\right) ; \mathrm{TxR}$ ot $=$ taxa de rotatividade em número de indivíduos $\left(\%\right.$.ano $\left.{ }^{-1}\right) ; \mathrm{N}=$ número de registro no Herbário Lages da Universidade do Estado de Santa Catarina (LUSC). Para os valores totais, em uma mesma taxa demográfica, letras distintas entre as áreas indicam diferenças $(p<0,05)$ de acordo com o teste de Kruskal-Wallis, seguido pelo teste de comparações múltiplas não paramétrico. Em taxas não distintas entre as áreas, pelos mesmos testes, não foram colocadas letras.

Tabela 3 - Teste qui-quadrado aplicado a uma tabela de contingência indicando o número de espécies observado (valores fora dos parênteses) e esperado (dentro) para as diferentes tendências de dinâmica do componente arbustivo-arbóreo regenerante $(\uparrow=$ incremento populacional, $\leftrightarrow$ $=$ estabilidade, $\downarrow=$ decremento), considerando as diferentes guildas de regeneração, em áreas campestres alto-montanas no Parque Nacional de São Joaquim, SC.

Table 3 - Chi-square test applied to a contingency table indicating the number of observed (values outside parentheses) and expected (inside) species for different dynamic trends of regenerating a treeshrub component $(\uparrow=$ populational increment, $\leftrightarrow=$ stability, $\downarrow=$ decrement), considering different regeneration guilds, in upper-montane grassland areas at São Joaquim National Park, SC state.

\begin{tabular}{|c|c|c|c|c|c|}
\hline & \multirow{2}{*}{$\begin{array}{l}\text { Tendências } \\
\text { de dinâmica }\end{array}$} & \multicolumn{3}{|c|}{ Guildas de regeneração } & \multirow{2}{*}{ Total } \\
\hline & & $\mathbf{P}$ & CEL & CTS & \\
\hline \multirow{4}{*}{ Área 1} & $\uparrow$ & $4(5,19)$ & $9(6,92)$ & $2(2,88)$ & 15 \\
\hline & $\leftrightarrow$ & $3(3,12)$ & $3(4,15)$ & $3(1,73)$ & 9 \\
\hline & $\downarrow$ & $2(0,69)$ & $0(0,92)$ & $0(0,38)$ & 2 \\
\hline & Total & 9 & 12 & 5 & 26 \\
\hline \multirow{4}{*}{ Área 2} & $\uparrow$ & $4(4,17)$ & $4(3,48)$ & $0(0,35)$ & 8 \\
\hline & $\leftrightarrow$ & $5(6,26)$ & $6(5,22)$ & $1(0,52)$ & 12 \\
\hline & $\downarrow$ & $3(1,57)$ & $0(1,30)$ & $0(0,13)$ & 3 \\
\hline & Total & 12 & 10 & 1 & 23 \\
\hline \multirow{4}{*}{ Área 3} & $\uparrow$ & 1 & 0 & 0 & 1 \\
\hline & $\leftrightarrow$ & 2 & 1 & 0 & 3 \\
\hline & $\downarrow$ & 0 & 0 & 0 & 0 \\
\hline & Total & 3 & 1 & 0 & 4 \\
\hline
\end{tabular}

Em que: $\mathrm{P}=$ pioneiras, $\mathrm{CEL}=$ clímax exigentes em luz, $\mathrm{CTS}=$ clímax tolerantes à sombra.

Área 1: $\mathrm{X}^{2}=6,2015 ; p=0,1846$; Área 2: $\mathrm{X}^{2}=3,9931 ; p=0,4069$. 
As cinco espécies mais abundantes foram Baccharis uncinella (P), Solanum cassioides (P), Solanum paranense (P), Myrceugenia regnelliana (CEL) e Berberis laurina (P) na Área 1; e Baccharis uncinella $(\mathrm{P})$, Campovassouria cruciata $(\mathrm{P})$, Senecio brasiliensis $(\mathrm{P})$, Croton cf. patrum $(\mathrm{P})$ e Solanum paranense $(\mathrm{P})$ na Área 2. Na Área 3, as únicas espécies arbustivo-arbóreas presentes foram Siphoneugena reitzii $(\mathrm{CEL})$, Baccharis uncinella $(\mathrm{P})$, Gaylussacia angustifolia $(\mathrm{P})$ e Baccharis nummularia $(\mathrm{P})$. Dentre as espécies mais abundantes na Área 1, todas apresentaram mudança líquida positiva, com destaque para Solanum cassioides e Berberis laurina, com aumento de 37,24\% e 31,71\%, respectivamente, nos tamanhos populacionais. Na Área 2, Solanum paranense e Croton cf. patrum se destacaram por apresentarem, respectivamente, os maiores valores de mudança líquida positiva $\left(88,00 \%\right.$.ano $\left.0^{-1}\right)$ e negativa $(-32,61 \%$. $\left.\mathrm{ano}^{-1}\right)$. Na Área 3, Baccharis nummularia, que era inexistente em 2014, foi a única espécie que apresentou recrutamento, com apenas dois indivíduos.

Figura 1- Relações entre as taxas demográficas $($ TxMor $=$ taxa de mortalidade; TxRec $=$ taxa de recrutamento; $T \times M L=$ taxa de mudança líquida e $T x R o t=$ taxa de rotatividade), do componente arbustivo-arbóreo regenerativo em três áreas estudadas no Parque Nacional de São Joaquim, SC. A parte inferior da diagonal apresenta os gráficos de dispersão entre os valores das taxas por subparcela e a parte superior da diagonal apresenta as correlações e as significâncias das correlações entre as taxas.

Figure $1-$ Relationships among demographic rates $(\mathrm{TxMor}=$ mortality rate; TxRec $=$ recruitment rate; $\mathrm{TxML}=$ net change rate and $\mathrm{TxR}$ ot $=$ turnover rate), of regenerating tree-shrub component in three studied area at São Joaquim National Park, SC state. The inferior part of diagonal presents the scatterplots between sub-plots rate values and superior part of diagonal shows the correlations between rates and its significance.

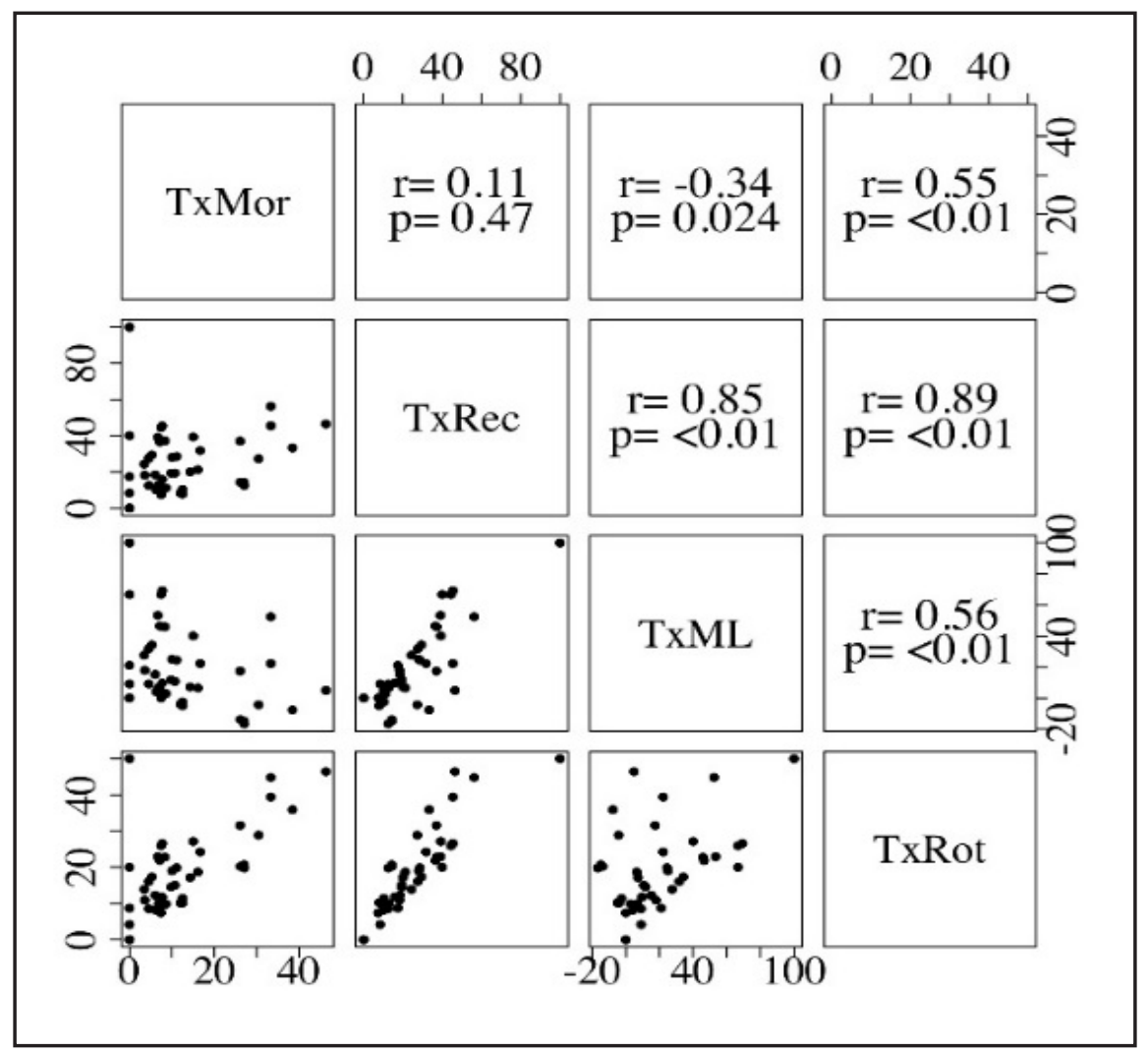


Figura 2 - Análise de Componentes Principais com variáveis ambientais das subparcelas de três áreas estudadas no Parque Nacional de São Joaquim, SC. Argila $=$ teor de argila; silte $=$ teor de silte; bases = saturação por bases; $\mathrm{Mg}=$ teor de magnésio $; \mathrm{pH}=$ valor de $\mathrm{pH} ; \mathrm{Na}=$ teor de sódio; $P=$ teor de fósforo; pen.med $=$ resistência à penetração média; pen.max $=$ resistência à penetração máxima; pen20 $=$ resistência à penetração a $20 \mathrm{~cm} ; \mathrm{K}=$ teor de potássio; $C$ TCef $=C T C$ efetiva; areia = teor de areia; $\mathrm{H} . \mathrm{Al}=$ índice $\mathrm{H}+\mathrm{Al} ; \mathrm{MO}=$ teor de matéria orgânica; $\mathrm{Al}=$ teor de alumínio; cota $=$ cota altitudinal; dec.med $=$ declividade média; Sal $=$ saturação por alumínio.

Figure 2 - Principal component analysis of environmental variables from sub-plots of three studied areas at São Joaquim National Park, SC state. Argila = clay content; silte= silt content; bases = bases saturation; $\mathrm{Mg}=$ magnesium content; $\mathrm{pH}=\mathrm{pH}$ values; $\mathrm{Na}=$ sodium values; $\mathrm{P}=$ phosphorus content; pen.med $=$ mean penetration resistance; pen. $\max =$ maximum penetration resistance; pen 20 = penetration resistance at $20 \mathrm{~cm}$ deep; $\mathrm{K}=$ potassium content CTCef $=$ effective cation exchange capacity; areia $=$ sand content; $\mathrm{H} . \mathrm{Al}=\mathrm{H}+\mathrm{Al}$ index; $\mathrm{MO}=$ organic matter content; $\mathrm{Al}=$ aluminum content; $\operatorname{cota}=$ elevation; dec. med = mean declivity; Sal = aluminium saturation .

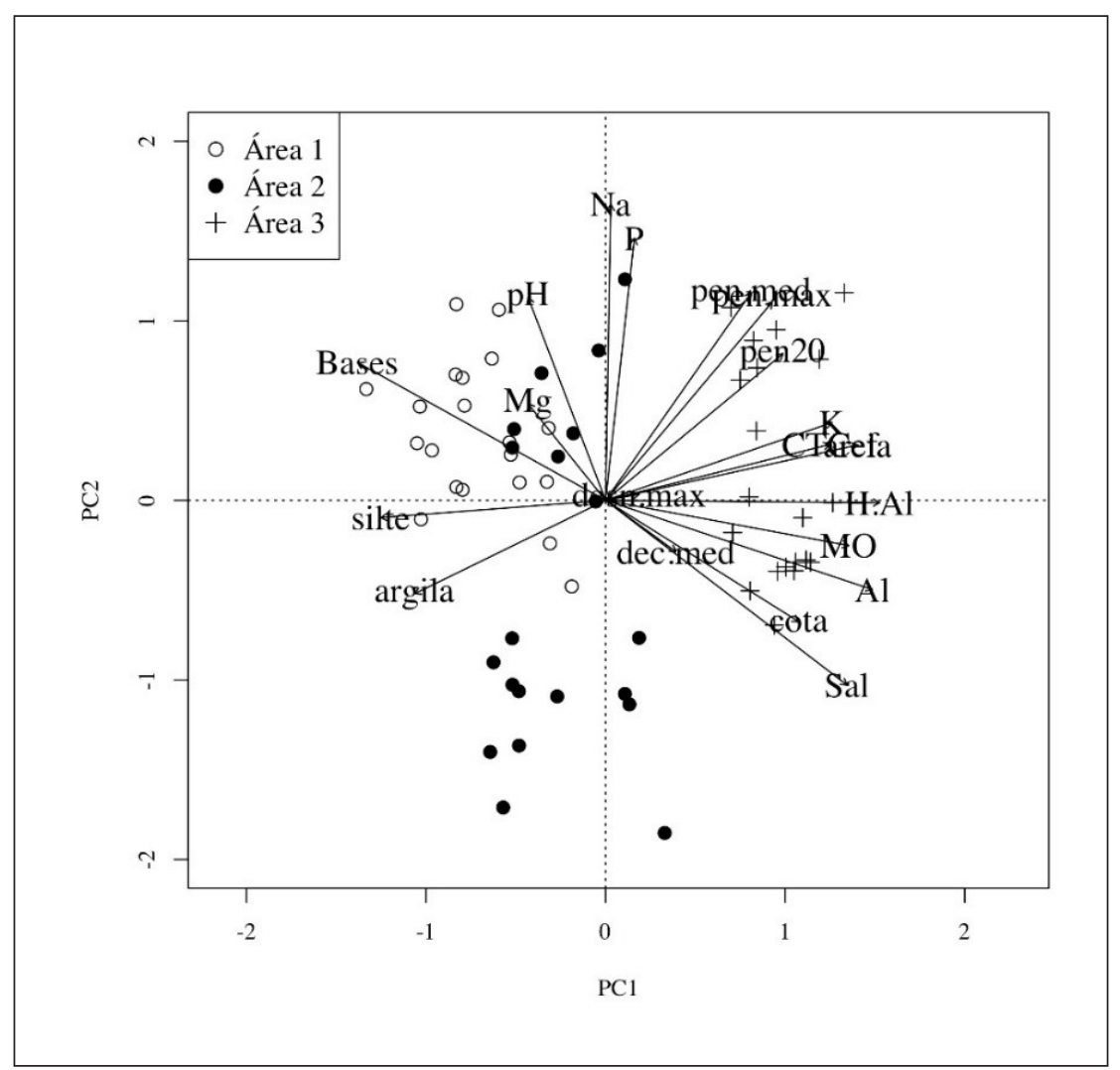


Em todas as áreas, a maioria das espécies apresentou tendência de incremento ou estabilidade populacional, porém, sem relação com as guildas de regeneração $(p>0,05)$ (Tabela 3). Assim, todas as guildas de regeneração apresentaram as mesmas proporções de espécies com incremento, estabilidade ou decremento populacional.

Ao se analisar as relações entre as taxas demográficas, considerando as três áreas, observa-se que não existiu relação significativa entre a mortalidade e o recrutamento $(\mathrm{r}=0,11 ; p=0,47)$ (Figura 1), indicando que estas taxas ocorreram de forma espacialmente dissociadas. A taxa de mudança líquida foi influenciada, principalmente, pelo recrutamento $(\mathrm{r}=0,85 ; p<0,01)$, o que explica o incremento de abundância nas áreas; e os locais com maior rotatividade foram aqueles com maior mortalidade $(\mathrm{r}=0,55$; $p<0,01)$ e recrutamento $(\mathrm{r}=0,89 ; p<0,01)$.A PCA (Figura 2$)$ indicou que o Eixo 1 explicou a maior proporção da variação ambiental observada nas três áreas $(41,58 \%)$, e este explicou de forma significativa apenas a taxa de recrutamento (Tabela 4). As variáveis que apresentaram maiores correlações com este eixo da PCA foram o índice de $\mathrm{H}+\mathrm{Al}(0,32)$, o teor de alumínio (Al) $(0,32)$ e a soma de bases $(-0,29)$. Assim, observa-se que, da esquerda para a direita na ordenação produzida pela PCA (PCA1), ocorre uma redução na fertilidade do solo, de parcelas com maior soma de bases e menores valores de índice $\mathrm{H}+\mathrm{Al}$ e teor de Al, para parcelas com menos bases e maiores valores de Al. Quanto maiores os valores de PCA1 (direita do gráfico), menores foram as taxas de recrutamento, indicando limitação da regeneração nesses locais, como nas parcelas da Área 3, localizadas no extremo à direita.

Tabela 4 - Modelos Lineares Mistos das taxas demográficas (TxMor = taxa de mortalidade; TxRec = taxa de recrutamento; $T \times M L=$ taxa de mudança líquida em número de indivíduos e TxRot $=$ taxa de rotatividade em número de indivíduos) do componente arbustivo-arbóreo regenerante em áreas campestres alto-montanas no Parque Nacional de São Joaquim, SC, em função do gradiente definido pelo Eixo 1 da PCA ambiental.

Table 4 - Mixed Linear Models of demographic rates $(\mathrm{TxMor}=$ mortality rate; TxRec $=$ recruitment rate; $T x M L=$ net change rate in number of individuals and $T x R o t=$ rate of turnover in number of individuals) of the shrub-tree component in upper montane grassland areas in São Joaquim National Park, SC state, in function of the gradient defined by the environmental axis of PCA.

\begin{tabular}{|c|c|c|c|c|}
\hline & PCA1 & $p$ & Efeito aleatório & Estrutura da variância \\
\hline TxMor & 2,95 & 0,254 & Intercepto & varIdent \\
\hline $\log (\mathrm{TxRec})$ & $-0,61$ & 0,008 & Intercepto & varExp \\
\hline TxML & $-13,56$ & 0,199 & Intercepto & $\begin{array}{l}\text { varComb(varIdent, } \\
\text { varFixed) }\end{array}$ \\
\hline TxRot & $-3,39$ & 0,401 & Intercepto & varIdent \\
\hline
\end{tabular}

Em que: $p=$ significância da variável explicativa (PCA1) sobre as taxas demográficas. *Funções com o propósito de incorporar a estrutura da variância aos modelos, onde VarIdent: permite a heterogeneidade do resíduo em função das diferentes áreas; varExp: permite a heterogeneidade do resíduo a partir de uma função exponencial da variância de PCA1; varFixed: permite a heterogeneidade do resíduo à medida que ocorre o aumento dos valores de PCA1. VarComb: combinação de duas estruturas da variância. 


\section{Discussão}

Os resultados demonstraram que, no período de estudo, após sete (Áreas 2 e 3) e oito (Área 1) anos da proteção integral contra distúrbios de origem antrópica, o processo de regeneração natural do componente arbustivo-arbóreo nos campos alto-montanos foi caracterizado pelo incremento na abundância de indivíduos, estabilidade na riqueza e também estabilidade na proporção da participação das espécies nas guildas de regeneração. Também houve independência espacial entre a mortalidade e o recrutamento e limitação do recrutamento em função da baixa fertilidade natural do solo e elevada disponibilidade de Al. Apesar dos padrões em comum, observou-se que o processo de expansão florestal no campo nativo (Área 3) ocorreu de forma mais lenta do que o processo de sucessão florestal secundária nos campos antrópicos (Áreas 1 e 2), sendo este aspecto parcialmente explicado pelas diferenças nas condições edáficas.

$\mathrm{O}$ aumento na abundância de indivíduos se deu, principalmente, em função do incremento do tamanho populacional de espécies pioneiras, o que indica que as áreas ainda se encontram em fase inicial de sucessão, uma vez que este é um padrão desta fase sucessional (SWAINE; HALL, 1983; VAN BREUGEL; BONGERS; MARTÍNEZ-RAMOS, 2007). A manutenção da riqueza (ganho de apenas uma espécie nas Áreas 1 e 3) e a estabilidade da fase sucessional reforçam a ideia de que o processo de reorganização florística pode não ocorrer de forma contínua (VAN BREUGEL; BONGERS; MARTÍNEZ-RAMOS, 2007) e pode ocorrer de forma mais lenta do que a reorganização estrutural.

Apesar deste padrão geral, observou-se que, para o período considerado, a dinâmica não ocorreu de forma homogênea entre as áreas, com a Área 2 apresentando maior taxa de mortalidade. Além de supostas influências relacionadas às diferenças ambientais (e.g. altitude), estas variações no padrão de dinâmica podem estar relacionadas com diferenças no grau de desenvolvimento do estágio sucessional entre as áreas, uma vez que a maior representatividade de espécies e indivíduos pioneiros na Área 2 sugere um grau de estágio sucessional mais inicial neste local. De fato, a Área 2 foi integralmente protegida mais recentemente (2008) do que a Área 1 (2007). Considerando que espécies mais iniciais apresentam usualmente um ciclo de vida mais curto, uma maior taxa de mortalidade deste grupo tem sido relatada na literatura (VAN BREUGEL; BONGERS; MARTÍNEZ-RAMOS, 2007).

A independência entre a mortalidade e o recrutamento indica que, assim como em florestas em avançado em estágio de sucessão (e.g., SALAMI et al., 2014), a dinâmica do componente arbóreo não é um processo espacialmente homogêneo, sugerindo a existência de fatores ecológicos, bióticos e, ou, abióticos influenciando as taxas demográficas do componente regenerativo.

Arelação significativa entre a taxa de recrutamento e a fertilidade do solo(relação positiva) e também com os teores de $\mathrm{H}+\mathrm{Al}$ e Al (relação negativa), sintetizada pelo Eixo 1 da PCA ambiental, sugere que a disponibilidade de nutrientes e o excesso por Al representam fatores determinantes para o estabelecimento de florestas em áreas de campos alto-montanos no sul do Brasil, atuando como filtros ambientais. De fato, Marcon et al. (2014), avaliando a influência de gradientes ambientais sobre a organização de uma FOM Alto-Montana no mesmo município, observaram elevada substituição florística em função da fertilidade do solo e topografia, o que reforça a ideia destes fatores na organização de comunidades arbóreas em elevada altitude. Da mesma forma, Moraes et al. (2016), avaliando a organização de comunidades de plantas em áreas campestres no Paraná a partir de atributos funcionais, concluíram que os aspectos edáficos relacionados à fertilidade natural do solo foram determinantes. Assim, na região sul do Brasil, além do clima (JESKE-PIERUSCHKA et al., 2013), do regime de distúrbios (OVERBECK et al., 2007), da presença de elementos nucleadores (DUARTE et al., 2006; CARLUCCI; DUARTE; PILLAR, 2011; CARLUCCI et al., 2011; MARCILIO-SILVA et al., 2015), da distância da borda florestal (MÜLLER et al., 2012) e da predação de sementes (IOB; VIEIRA, 2008), o presente estudo corrobora a ideia de que o estabelecimento da vegetação arbustivo-arbórea também seja influenciado pela disponibilidade de nutrientes (SOBANSKI; MARQUES, 2014) e excesso de Al no solo. Ressalta-se que a Área 3, de campo natural, na qual foram observados poucos indivíduos arbustivo-arbóreos, destacou-se por apresentar os solos de menor fertilidade e maiores teores de Al, o que contribuiu para sustentar esta suposição. 


\section{Conclusões}

Conclui-se que, após a proteção integral recente das áreas campestres contra distúrbios de origem antrópica, o processo de regeneração natural do componente arbustivo-arbóreo no período de estudo ainda se encontra em fase inicial, caracterizado pela elevada participação de espécies iniciais de sucessão, pelo incremento do número de indivíduos e estabilidade na riqueza. O padrão de dinâmica variou entre as áreas em função da fase de cada área ao longo da trajetória sucessional e da natureza do campo (natural ou antrópico), embora, durante o período de avaliação, tenha sido observada estabilidade na proporção da participação das espécies nas guildas sucessionais dentro de cada área. Ainda, o recrutamento de novos indivíduos foi limitado pela disponibilidade de nutrientes no solo e elevado teor de Al. Desta forma, os resultados encontrados sugerem que o processo de expansão florestal em campos nativos é também limitado por condições edáficas, ocorrendo, assim, de forma mais lenta do que sucessão florestal em campos antrópicos.

\section{Agradecimentos}

À CAPES, pela concessão de bolsa à primeira autora, e ao CNPq, pela concessão de bolsa de produtividade para o segundo e o terceiro autores e pelo financiamento do projeto. Ao Instituto Chico Mendes de Conservação da Biodiversidade (ICMBio). À equipe do PARNA de São Joaquim, em especial ao M.Sc. Michel Omena. Aos pesquisadores Dr. Marcos Eduardo Guerra Sobral e Dr. Cláudio Augusto Mondin, pelo auxílio na identificação do material botânico.

\section{Referências}

BEHLING, H.; PILLAR, V. D. Late quaternary vegetation, biodiversity and fire dynamics on the southern brazilian highland and their implication for conservation and management of modern araucaria forest and grassland ecosys tems. Philosophical Transactions of the Royal Society B, London, v. 362 , n. 1478 , p. $243-251,2007$.

BEHLING, H. et al. Late-Holocene fire history in a forest-grassland mosaic in southern Brasil: implications for conservation. Applied Vegetation Science, Hoboken, v. 10, n. 1, p. 81-90, 2007.

CARLUCCI, M. B.; DUARTE, L. S.; PILLAR, V. D. Nurse rocks influence forest expansion over native grassland in southern Brazil. Journal of Vegetation Science, Hoboken, v. 22, n. 1, p. 111-119, 2011.

CARLUCCI, M. B. et al. Edge expansion of Araucaria forest over southern Brazilian grasslands relies on nurse plant effect. Community Ecology, Budapest, v. 12, n. 2, p. 196-201, 2011.

DUARTE, L. S. et al. Role of nurse plants in Araucaria Forest expansion over grassland in south Brazil. Austral Ecology, Hoboken, v. 31, n. 4, p. 520-528, 2006.

HIJMANS, R. J. et al. Very high resolution interpolated climate surfaces for global land areas. International Journal of Climatology, Chichester, v. 25, n. 15, p. 1965-1978, 2005.

IBGE. Manual técnico da vegetação brasileira. Rio de Janeiro: Fundação Instituto Brasileiro de Geografia e Estatística, 2012. 271 p.

IOB, G.; VIEIRA, E. M. Seed predation of Araucaria angustifolia (Araucariaceae) in the Brazilian Araucaria Forest: influence of deposition site and comparative role of small and 'large' mammals. Plant Ecology, New York, v. 198, n. 2, p. 185-196, 2008.

JESKE-PIERUSCHKA, V. et al. New insights into vegetation, climate and fire history of southern Brazil revealed by a 40,000 year environmental record from the State Park Serra do Tabuleiro. Vegetation History and Archaeobotany, New York, v. 22, n. 4, p. 299-314, 2013. 
KORNING, J.; BALSLEV, H. Growth and mortality of trees in Amazonian tropical rain forest in Ecuador. Journal of Vegetation Science, Hoboken, v. 5, n. 1, p. 77-86, 1994.

MARCILIO-SILVA, V. et al. Nurse abundance determines plant facilitation networks of subtropical forest-grassland ecotone. Austral Ecology, Hoboken, v. 40, n. 8, p. 898-908, 2015.

MARCON, A. K. et al. Variação florístico-estrutural em resposta à heterogeneidade ambiental em uma floresta nebular em Ububici, Planalto Catarinense. Scientia Forestalis, Piracicaba, v. 42, n. 103, p. 439-450, 2014.

MORAES, D. A. et al. Edaphic filters and the functional structure of plant assemblages in grasslands in southern Brazil. Journal of Vegetation Science, Hoboken, v. 27, n. 1, p. 100-110, 2016.

MÜLLER, S. C. et al. Plant functional types of woody species related to fire disturbance in forest-grassland ecotones. Plant Ecology, New York, v. 189, n. 1, p. 1-14, 2007.

MÜLLER, S. C. et al. Woody species patterns at forest-grassland boundaries in southern Brazil. Flora, Jena, v. 207, n. 8, p. 586-598, 2012.

OLIVEIRA, J. M.; PILLAR, V. D. P. Vegetation dynamics on mosaics of Campos and Araucaria forest between 1974 and 1999 in Southern Brazil. Community Ecology, Budapest, v. 5, n. 2, p. 197-202, 2004.

OLIVEIRA FILHO, A. T. et al. Differentiation of streamside and upland vegetation in an area of montane semideciduous forest in southeastern Brazil. Flora, Jena, v. 189, n. 4, p. 287-305, 1994a.

OLIVEIRA FILHO, A. T. et al. Dinâmica da comunidade e populações arbóreas da borda e interior de um remanescente florestal na Serra da Mantiqueira, Minas Gerais, em um intervalo de cinco anos (1999-2004). Revista Brasileira de Botânica, São Paulo, v. 30, n. 1, p. 149-161, 2007.

OLIVEIRA FILHO, A. T. et al. Effects of soils and topography on the distribution of tree species in a tropical riverine forest in south-eastern Brazil. Journal of Tropical Ecology, Cambridge, v. 10 , n. 4 , p. $483-508,1994$ b.

OVERBECK, G. E. et al. Brazil's neglected biome: the South Brazilian Campos. Perspectives in Plant Ecology, Evolution and Systematics, Amsterdam, v. 9, n. 2, p. 101-116, 2007.

R DEVELOPMENT CORE TEAM. R: a language and environment for statistical computing. [2015]. Disponível em: <http://www.R-project.org> Acesso em: 08 out. 2015.

SALAMI, B. et al. Influência de variáveis ambientais na dinâmica do componente arbóreo em um fragmento de Floresta Ombrófila Mista em Lages, SC. Scientia Forestalis, Piracicaba, v. 42, n. 102, p. 197-207, 2014.

SHEIL, D.; JENNINGS, S.; SAVILL, P. Long-term permanent plot observations of vegetation dynamics in Budongo, a Ugandan rain forest. Journal of Tropical Ecology, Cambridge, v. 16, n. 6, p. 865-882, 2000 .

SILVA, L. C. R.; ANAND, M. Mechanisms of Araucaria (Atlantic) forest expansion into southern Brazilian grasslands. Ecosystems, Nova York, v. 14, n. 8, p. 1354-1371, 2011.

SOBANSKI, N.; MARQUES, M. C. M. Effects of soil characteristics and exotic grass cover on the forest restoration of the Atlantic Forest region. Journal for Nature Conservation, Amsterdam, v. 22, n. 3, p. 217-222, 2014.

SWAINE, M. D.; HALL, J. B. Early succession on cleared forest land in Ghana. Journal of Ecology, London, v. 71, n. 2, p. 601-627, 1983.

SWAINE, M. D.; WHITMORE, T. C. On the definition of ecological species groups in tropical rain forest. Vegetatio, New York, v. 75, n. 1/2, p. 81-86, 1988.

VAN BREUGEL, M.; BONGERS, F.; MARTÍNEZ-RAMOS, M. Species dynamics during early secondary forest succession: recruitment, mortality and species turnover. Biotropica, Hoboken, 
Dallabrida, J. P.; Silva, A.; Higuchi, P.; Larsen, J. G.; Santos, G. N.; Lima, C. L.; Rodrigues, L. D.; Machado, F. D.; Cruz, A. P.; Nunes, A. S.

v. 39, n. 5, p. 610-619, 2007.

ZUUR, A. et al. Mixed effects models and extensions in ecology with R. Amsterdam: Springer Science \& Business Media, 2009. 574 p. 